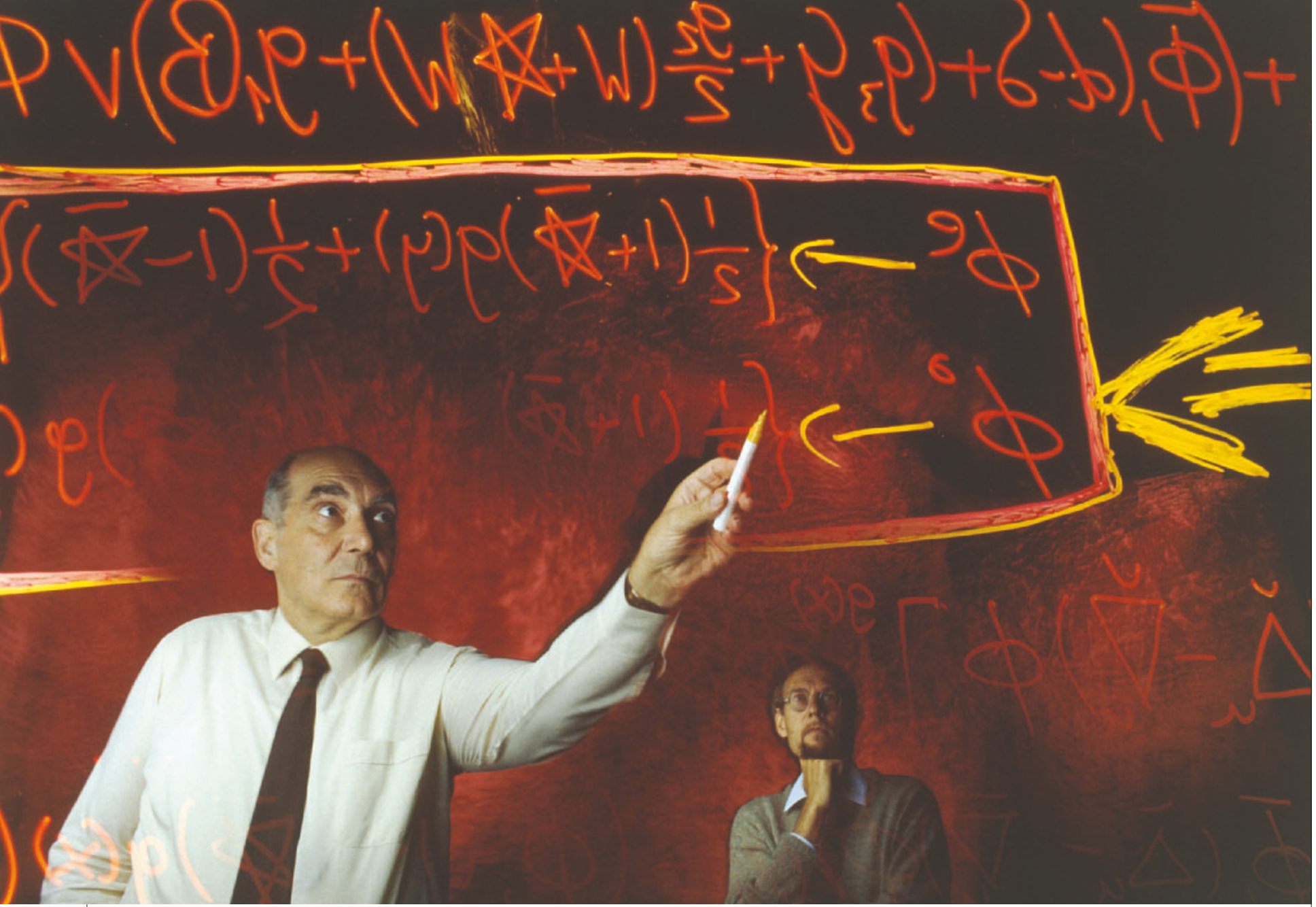

\title{
So, what's your theory?
}

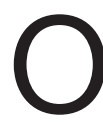
ne hundred years ago, when Albert Einstein penned his era-defining papers on brownian motion, the photoelectric effect and special relativity, he was just 26 years old. Reaching scientific greatness at such a young age was exceptional then and may be even harder today. But when looking at physics in the twenty-first century, there's still much to discover by asking a young physicist: what's your theory?

In the following pages, Nature offers a glimpse into the lives of four young theorists (all under 35) who are making waves in their chosen fields.

In Einstein's youth, the focus of theoretical physics was in Europe: Niels Bohr was in Copenhagen, Max Planck was in Berlin. Today it is harder to find the centre of the theoretical universe - collaborative research is increasingly international, and most theorists, who need little more than a laptop, can work anywhere.

But it seems that many young theorists opt to spend their formative years in the United States. Although US enrolment of foreign graduate students has fallen in recent years, they still make up about half of the total in physics, and of these some $40 \%$ are theorists. Not all of these students will stay in the United States - many, including three featured here, will head for good positions back home.

All of our interviewees share a willingness to push big ideas forwards while also asking how - and how soon they can be tested. Einstein had to wait just a few years for predictions from his 1915 general theory of relativity to be confirmed by observations of a solar eclipse. But many theorists finish their careers without seeing any experimental check on their ideas. Our young theorists not only know that they must think big, but also that they must pit their wildest theories against reality.

Over the next few years, many theorists will be directing their attention to the world's largest particle accelerator, currently being built near Geneva in Switzerland, for experimental confirmation of their ideas. Here, physicists hope to find some support for exotic notions such as extra dimensions (see 'In search of hidden dimensions', page 10). Elsewhere, missions such as the Planck satellite will provide data on the early Universe that may help to shore up theories about the moment of creation (see 'The long-distance thinker', page 12). The hoped-for construction of the first quantum computer would test fundamental ideas about the quantum world (see 'A theorist of errors', page 9), whereas the creation of exotic materials is continually pushing our knowledge of electron behaviour (see 'Can electrons do the splits?', page 11).

As we look back at the past 100 years and celebrate the World Year of Physics in 2005, there are still plenty of twentieth-century puzzles that theorists would like to
Young star: Einstein was only 26 when he published three seminal papers. complete, but there is also the tantalizing possibility that something totally unknown is just around the corner. It will be young theorists such as those featured here who will tackle these challenges. Sarah Tomlin edits News Features for Nature from New York. 